\title{
Auxotrophic mutations of Trichophyton rubrum created by in vitro synthesized Cas9 ribonucleoprotein
}

\author{
Oliver Blechert', Huan Mei ${ }^{1}$, Xiaohui Zang ${ }^{1}$, Hailin Zheng ${ }^{1,2}$, Guanzhao Liang ${ }^{1}$ and Weida Liu ${ }^{1,2,3^{*}}$
}

\begin{abstract}
Background: Trichophyton rubrum is an obligate human parasitic fungus and responsible for approximately 80-90\% of dermatomycosis in human. Molecular genetic manipulations of this pathogen are challenging and available tools and protocols are only rudimentary. We adapt molecular genetics methods of well established fungal model organism, to knock out genes in T. rubrum. For the adaptation, crucial modifications are necessary. With the implementation of in vitro synthesized Cas9-sgRNA ribonucleoprotein complex, it is possible to adapt molecular genetic methods, to knock out genes in T. rubrum.

Results: The gene knock-out method is based on integration of a selection marker into the target site, to interrupt the gene translation. The target gene gets preassigned by the homologous sequence of the in vitro synthesized Cas9-sgRNA ribonucleoprotein complex. To develop the method, we first isolated and characterized a T. rubrum strain with a high amount of microconidia. Next, we developed a transformation protocol, whereby the Cas9-sgRNA ribonucleoprotein gets delivered into the fungal protoplast by the PEG method.

We knocked out the URA3 gene and resulted, as predicted, uracil auxotrophic strains. These strains can be used for specific gene knock-outs by reintegrating the URA3 fragment and selection on uracil lacking cultivation media. Exemplary, we knocked out the TRP3 gene and got the predicted phenotype, tryptophan auxotrophic strains. The mutation had been verified by sequencing.

Conclusions: We developed a method, based on in vitro synthesized Cas9-sgRNA ribonucleoprotein complex, for target specific gene knock-outs in T. rubrum. We knocked out the Ura3 gene and resulted uracil auxotrophic strains. These strains were used for target specific gene knock-outs by reintegrating the Ura3 fragment into the target gene site to interrupt the gene transcription. The developed method allows to adapt sophisticate gene manipulation methods of model fungal species to non-model species.
\end{abstract}

Keywords: Trichophyton rubrum, Cas9 ribonucleoprotein complex, Gene knock-out, Uracil, Tryptophan

\section{Background}

Trichophyton rubrum (Castellani) Sabouraud 1911 is a haploid ascomycetes fungus of the order Onygenales. As an obligate human pathogenic fungus, T. rubrum is in the focus of medicine and research for more than a century. Moreover, in the last decades the fungus got the most relevant

\footnotetext{
* Correspondence: liumyco@hotmail.com

'Department of Medical Mycology, Institute of Dermatology, Chinese Academy of Medical Science and Peking Union Medical College, Nanjing, Jiangsu 210042, People's Republic of China

${ }^{2}$ Jiangsu Key Laboratory of Molecular Biology for Skin Diseases and STIs,

Nanjing, Jiangsu 210042, People's Republic of China

Full list of author information is available at the end of the article
}

dermatophyte and is responsible for $80-90 \%$ of the dermatomycosis cases. Contrary, only limited genetic informations are available and the development of genetic tools and protocols are far behind in comparison to other medical important fungi as Candida albicans and Aspergillus spp. [1].

In fungal model-organism auxotrophic selection-markers are a standard tool for selecting transformed colonies. Widely used is the orotidine-5'-monophosphate decarboxylase gene, in Saccharomyces cerevisiae with the standard name URA3. The URA3 gene is part of the uracil pathway and knock down of the gene causes an auxotrophy, which can be compensated by supplying uracil (Ura) or uridine (Uri). A special advantage of using the URA3 gene as

(c) The Author(s). 2020 Open Access This article is distributed under the terms of the Creative Commons Attribution 4.0 International License (http://creativecommons.org/licenses/by/4.0/), which permits unrestricted use, distribution, and 
selection-marker is the possibility of counter-selection by 5 fluoroorotic acid (FOA) [2]. S. cerevisiae and C. albicans $\Delta u r a 3$ strains can not grow in the absence of uracil, but can grow on FOA media, whereas the wild type can grow in the absence of uracil but not on FOA media.

Integration of a DNA fragment into the genome of a fungus takes place at a double string break (DSB) sites in a chromosome. These breaks can be caused randomly by UV radiation or restriction enzymes. In Trichophyton mentagrophytes the restriction enzyme mediated integration (REMI) had been used to tag the fungus with a GFP signal [3]. When a DNA fragment gets integrated into the genome, basically two different types of repair mechanisms are involved. The first is the homologous directed repair (HDR) mechanism of the fungus. For this, the integration fragment must be designed with sequences that are homologous to both flanking sites. The DSB is repaired by homologous recombination and the fragment will be integrated at the target site. In S. cerevisiae and $C$. albicans genome manipulation by homologous recombination is the most commonly applied method. Since in Trichophyton spp., DNA fragments are dominantly integrated by the non-homologous end joining (NHEJ) repair mechanism, and homologous integration is inhibited. By knocking out the KU80 gene, which is a key factor of NHEJ, the rate of homologous integration increased in $T$. mentagraphitis from lower than 3\% to approximately 70\% [4].

The Cas9 protein allows to induce target specific DSBs. The Cas9 protein is guided by a sgRNA fragment to the specific site. Commonly the sgRNA consist of a $17-20 \mathrm{bp}$ long protospacer region and a $80 \mathrm{bp}$ long tail. The tail has a specific loop structure which is required for the ribonucleoprotein complex formation. The protospacer region is responsible for docking to a specific site at the chromosome [5]. By changing the protospacer sequence, homologous to the genome target sequence, DSBs can be induced in nearly every gene. In the well studied fungal genus Aspergillus, the Cas9 gene had been expressed from an autonomous replication vector [6]. In fungal species, which are not so well studied, and especially when no autonomous replication vectors are available, the Cas9 can be delivered as in vitro synthesized ribonucleoprotein complex instead of expressing both components from vectors. The method had been applied to Mucor circinelloides [7] and Fusarium oxysporum [8] and the ribonucleoprotein complex had been delivered into the cell by the PEG method.

In this study, we developed for the first time a transformation system for T. rubrum, based on delivering the Cas9-sgRNA ribonucleoprotein complex together with the URA3 gene DNA fragment into the protoplast by the PEG method. For this, we have isolated and characterized a suitable monoclonal wild-type strain and knocked out the URA3 gene. One of the $\triangle$ ura3 strains had been used to create a target specific knock-out based on the uracil selection method.

\section{Results}

\section{Fungal strains}

The two fungal strains, STRB008 and STRB012 were derived from clinical strains. After two rounds of single colony selection, the strains were preserved in September 2017 at $-80^{\circ} \mathrm{C}$. We tested the storage in $20 \%$ glycerol solution and the storage of conidia suspension dropped on filter disks. With both methods the fungi were recovered after one year, even after repeated freeze and thaw cycles.

The fungi were identified by phenotype, microscopic analysis and comparison of the internal transcribed spacer (ITS) sequence. On PDA plates STRB012 developed the typical red pigments (Fig. 1a, b), whereas on SC medium the fungus had a yellow to brownish color (Fig. 1c, d).
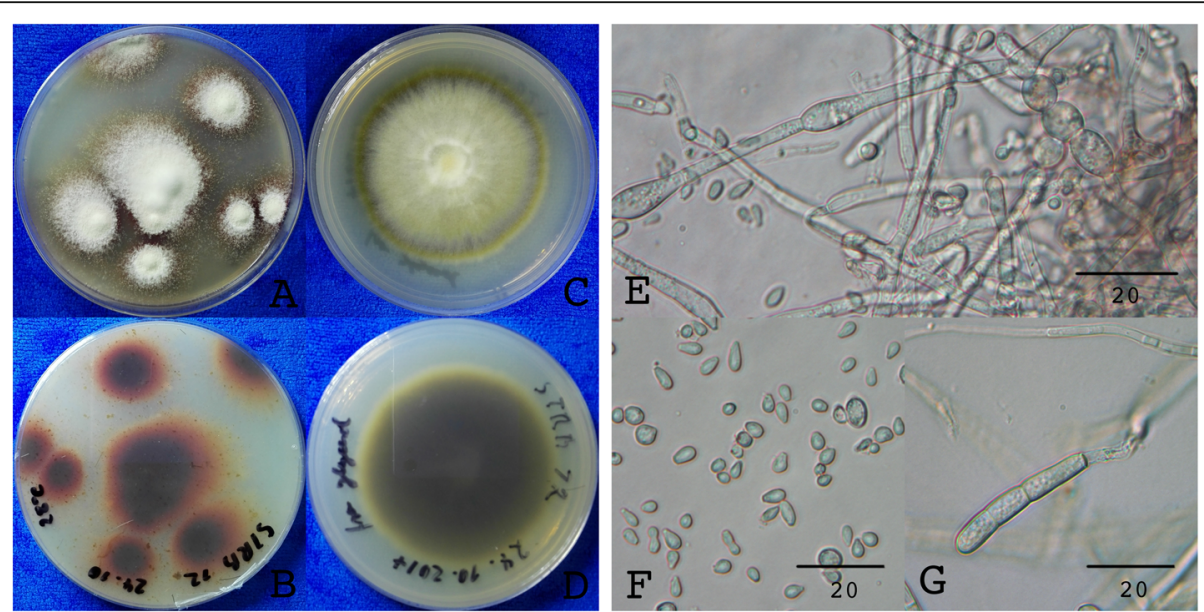

Fig. 1 Phenotype and morphology of T. rubrum STRB012. a, b. Three weeks old culture grown on PDA agar. The red pigmentation is strongly developed. $\mathbf{c}$, $\mathbf{d}$ Three weeks old culture grown on SC agar. The colony has a yellow to brownish pigmentation. e. Hyphae, microconidia and chlamydospores. f Microconidia. g Macroconidium 
Hyphae of STRB012 were septate, 3.5-4 $\mu \mathrm{m}$ in diameter, infrequent curved and typically branched in right angle. Chlamydospores were in short chains, rounded to oval and $6-10 \times 7-8 \mu \mathrm{m}$ (Fig. 1e). The microconidia were rounded to pear-shaped, flattened at the narrowed side and $4-6 \times 2-3 \mu \mathrm{m}$ (Fig. 1f). The macroconidia were septate, predominately in three cells, with a size of $28-45 \times 4-$ $5 \mu \mathrm{m}$ (Fig. 1g). Several microconidia developed two weeks after incubation at $28^{\circ} \mathrm{C}$ on $\operatorname{Tr} 1$ medium. The development of microconidia could be stimulated by increasing the $\mathrm{CO}_{2}$ level to $5 \%$ (Fig. 2a, b).

The ITS1 and 2 sequences were identical to the $T$. rubrum CBS 392.58, except of one nucleotide in strain STRB012. The ITS 1 sequence of CBS 392.58 (NCBI NR_131330.1) has a cytosine at position 213 instead of thymine (see Additional file 1).

\section{Knock-out of the uracil pathway}

For each of the knock-out experiments, two petri dishes, with $\operatorname{Tr} 1$ medium, were inoculated with STRB012. The cultures had been incubated at $28{ }^{\circ} \mathrm{C}$ with $5 \% \mathrm{CO}_{2}$ for three weeks. Conidia were harvested by overlaying the plates with liquid SC medium and gently scratching the surface with a spreader. The conidia dispersions were filtrated through Miracloth to remove hyphal fragments. The purified conidia dispersion were filled up to $20 \mathrm{ml}$ and shaken at $28^{\circ} \mathrm{C}$ for $20 \mathrm{~h}$ with $120 \mathrm{rpm}$ to induce germination. Afterwards, the liquid media were removed by centrifuging with $13,523 \mathrm{~g}$ for $10 \mathrm{~min}$ and washed twice with $\mathrm{KCl}$ buffer. The conidia were resuspended in $5 \mathrm{ml}$ lysis buffer and incubated for $200 \mathrm{~min}$ at $28^{\circ} \mathrm{C}$ with shaking. The cell wall digested conidia were centrifuged for $10 \mathrm{~min}$ with $9391 \mathrm{~g}$ and washed twice with $\mathrm{KCl}$ buffer. A total amount of 10 million protoplasts were resuspended in $250 \mu \mathrm{l}$ sucrose buffer by carefully flicking the reaction cup.

Each $100 \mu \mathrm{l}$ of the protoplast dispersion were added to $35 \mu$ transformation solution, gently mixed and incubated for $10 \mathrm{~min}$ on ice. Afterwards, $1 \mathrm{ml}$ of PEG buffer was added, mixed gently by inverting the reaction cups and incubated in a water bath for $10 \mathrm{~min}$ at $28^{\circ} \mathrm{C}$. For every transformation plate $250 \mu \mathrm{l}$ were pipetted to $7.5 \mathrm{ml} 40^{\circ} \mathrm{C}$ warm overlay media, supplied with uracil and uridine, mixed by inverting and poured over SC medium plates. Next day, the plates were overlaid with SC medium containing FOA.

After two weeks of incubation at $28^{\circ} \mathrm{C}$, distinct colonies have formed on the transformation plates (Fig. 3a), whereas on the control plates only downy hyphae were visible (Fig. 3b). Hyphal fragments of 39 distinct colonies were inoculated to FOA plates and incubated for 3 weeks. All colonies were grown well and hyphal fragments of the border of the colonies were inoculated on $\mathrm{SC}$-uracil selection plates.

After two weeks of inoculation, no growth of the selected strains was observed on -uracil medium, whereas the parent strain, STRB012, was grown also in the absence of uracil. On SC medium, supplemented with uracil and uridine, the transformed strains had a similar growth rate and appearance as the parent strain (Fig. 3c-f).

Three strains were selected for the verification of the genotype and the target regions were sequenced. In each of the three strains the URA3 gene was mutated 3 to 4 bases upstream of the PAM sequence (Fig. 3g). One strains had a deletion of one nucleotide, one strain had an insertion of one nucleotide and the third had an insertion of 68 nucleotides. The surrounding regions up and downstream had no additional mutations.

Single clone colonies of the two strains with one base indels were isolated and preserved as STRB018 and STRB019 at $-80^{\circ} \mathrm{C}$. The transformation experiment was repeated and resulted a similar amount of transformed strains. Of strain STRB008, which is characterized by a lower amount of microconidia in comparison to STRB012, one uracil auxotrophic mutation had been created and a single clone was preserved as STRB020.

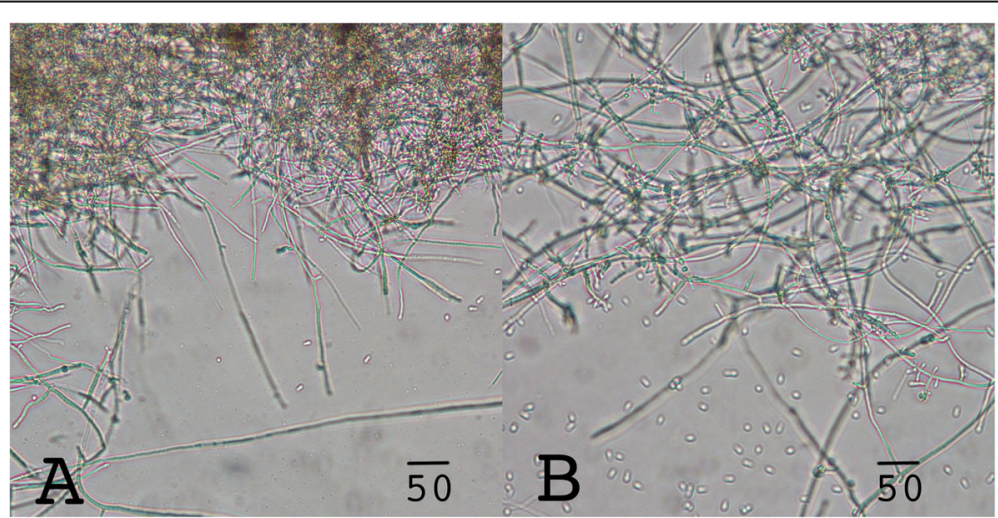

Fig. 2 Induction of microconidia by $\mathrm{CO}_{2}$. a Three weeks old culture grown on $\operatorname{Tr} 1$ media at $28^{\circ} \mathrm{C}$. G. same as $\mathbf{b}$ but supplied with $5 \% \mathrm{CO}_{2}$ to induce conidiogenesis 


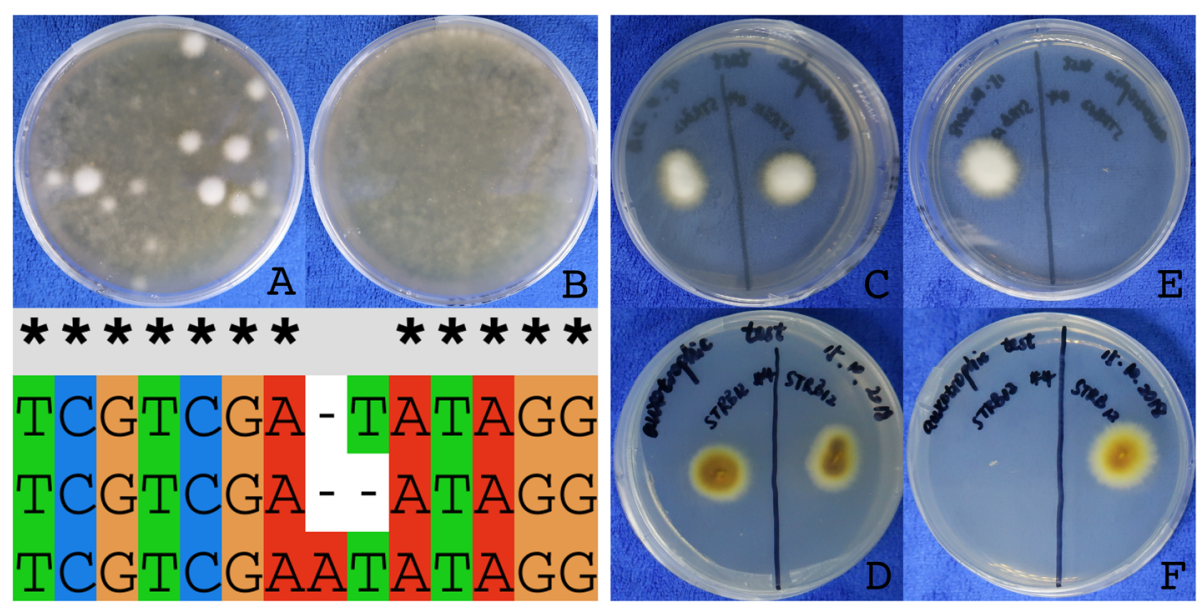

Fig. 3 Transformation and analysis of T. rubrum $\triangle$ ura3 strains. a Three weeks old transformation plate supplemented with FOA for counter selection. $\triangle$ ura3 strains are visible as distinct colonies. $\mathbf{b}$ Three weeks old control plate supplemented with FOA for counter selection. $\mathbf{c}$, d Auxotrophy test of $\triangle$ ura3 and parent strain. SC medium supplied with uracil and uridine. The wild-type and the $\Delta$ ura3 strain have a similar growth rate e, $\mathbf{f}$. Auxotrophy test of $\triangle$ ura3 and parent strain. SC medium without uracil and uridine. Only the wild-type strain is growing. $\mathbf{g}$ DNA sequence alignment of the mutated part of the URA3 gene. Top sequence: Part of the protospacer sequence including PAM sequence. Middle: Auxotrophic strain STRB018. Bottom: Auxotrophic strain STRB019

\section{Target specific knock-out of the TRP3 gene}

Next, we were using the $\Delta u r a 3$ strain STRB018 for creating tryptophan auxotrophic strains. We predicted TERG07826 as orthologous to the S. cerevisiae TRP3 gene and designed the primers and vectors. For knocking out the TRP3 gene, we modified the protocol. First, for the transformation and selection, we used SC medium without uracil. Second, the sgRNA has been transcribed from plasmid PTRB042 resp. 43 (Fig. 4). Further, $3 \mu \mathrm{g}$ URA3 fragments has been added to the

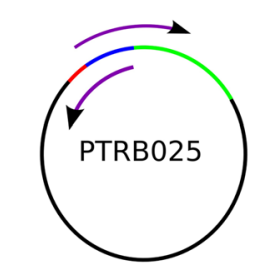

A

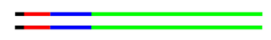

D

Legend

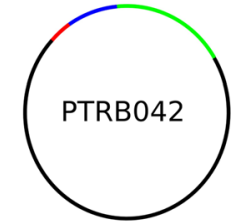

B

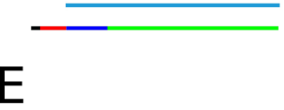

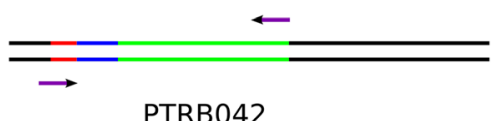

C

$\mathrm{F}$

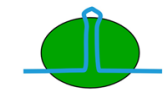

G

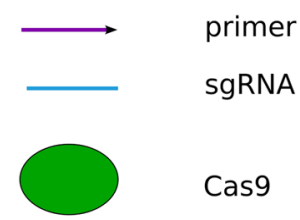

Fig. 4 Creation of TRP3 specific Cas9-sgRNA ribonucleoprotein complex. a Amplifying the plasmid PTRB025 with primer pair NTRB107/108 to change the protospacer region. Recirculate the DNA fragment with a Mutagenesis Kit. b Amplification of the modified plasmid in E. coli and verification of the modification by sequencing with M13 primers. $\mathbf{c}$ Linearize of the plasmid with Hindlll. $\mathbf{d}$. Amplification of the sgRNA template with primer pair NTRB80/81. e Transcription of the sgRNA with T7 RNA polymerase. $\mathbf{f}$ DNAse treatment and RNA purification. $\mathbf{g}$ Cas9-sgRNA ribonucleoprotein complex formation 
transformation solution. A total eight colonies appeared on the transformation plates (Fig. 5a, b). On the control plates, without adding Cas9-sgRNA ribonucleoprotein and the URA3 fragment, no colonies appeared even after one month of cultivation. All eight colonies were inoculated on SC plates without tryptophan and no growth had been observed, even after one month of inoculation (Fig. 5c, d). Both the wild-type strain STRB012 and the Aura3 strain STRB018, were growing on tryptophan lacking media.

Next, we amplified the Trp3 gene including the flanking region of all strains with the primer pair NTRB076/ 77 and got in six of the eight samples a single band (Table 1). We cloned four of DNA fragments into pUC19 plasmid for sequencing with the primers NTRB115 and 116. The inserted DNA fragments were in all cases at the predicted position 3-4 base pair upstream of the PAM sequence and in the flanking regions no further mutation occurred. In three of the four strains, the Ura3 DNA fragments were inserted into the Cas9 DSB sites. The fragment length of the original Ura3 PCR product was $2964 \mathrm{bp}$, but all inserted fragments were truncated. In one descendant the inserted fragment had lost one base at each of the ends. The lengths of the inserted Ura3 fragment of the two other
Table 1 Phenotype and length of the inserted marker fragment in the eight $\Delta$ trp3 strains

\begin{tabular}{|c|c|c|c|c|c|}
\hline \multirow[t]{2}{*}{ Strain } & \multicolumn{2}{|c|}{ Phenotype*1 $^{* 1}$} & \multicolumn{3}{|c|}{ Fragment length ${ }^{* 2}$} \\
\hline & Ura & Trp & $\overline{P C R^{* 3}}$ & & $\mathrm{red}^{* 4}$ \\
\hline STRB012 & $P$ & $P$ & NA & & NA \\
\hline STRB018 & A & $P$ & NA & & NA \\
\hline Atrp3_c1_1 & $P$ & A & $2130 \mathrm{bp}$ & + & $1955 \mathrm{bp}$ \\
\hline$\Delta \operatorname{trp3} 3$ c1_2 & $P$ & A & $270 \mathrm{bp}$ & + & $0 \mathrm{bp}^{* 5}$ \\
\hline Atrp3_c1_3 & $P$ & A & $2880 \mathrm{bp}$ & + & 2962 bp \\
\hline$\Delta \operatorname{trp3} 3$ c1_4 & $P$ & A & $0 \mathrm{bp}$ & - & NA \\
\hline$\Delta \operatorname{trp3} 3$ c1_5 & $P$ & A & $2370 \mathrm{bp}$ & + & $2380 \mathrm{bp}$ \\
\hline$\Delta \operatorname{trp3} 3$ c2_1 & $P$ & A & $1900 \mathrm{bp}$ & - & NA \\
\hline$\Delta \operatorname{trp3} 3$ c2_2 & $P$ & A & $990 \mathrm{bp}$ & - & NA \\
\hline$\Delta \operatorname{trp3} 3 \_223$ & $P$ & A & $2250 \mathrm{bp}$ & - & NA \\
\hline
\end{tabular}

${ }^{* 1}$ A: Auxotrophic, P: Prototrophic

*2 length of the inserted ura3 marker fragment

*3 calculated from agarose gel electrophoresis with the Lablmage Version 2.62 software. The last digit of the values were rounded up

$*^{4}$ counted from the Sanger sequencing result

${ }^{*}$ no ura3 fragment was inserted, but an unintentional bacterial DNA fragment
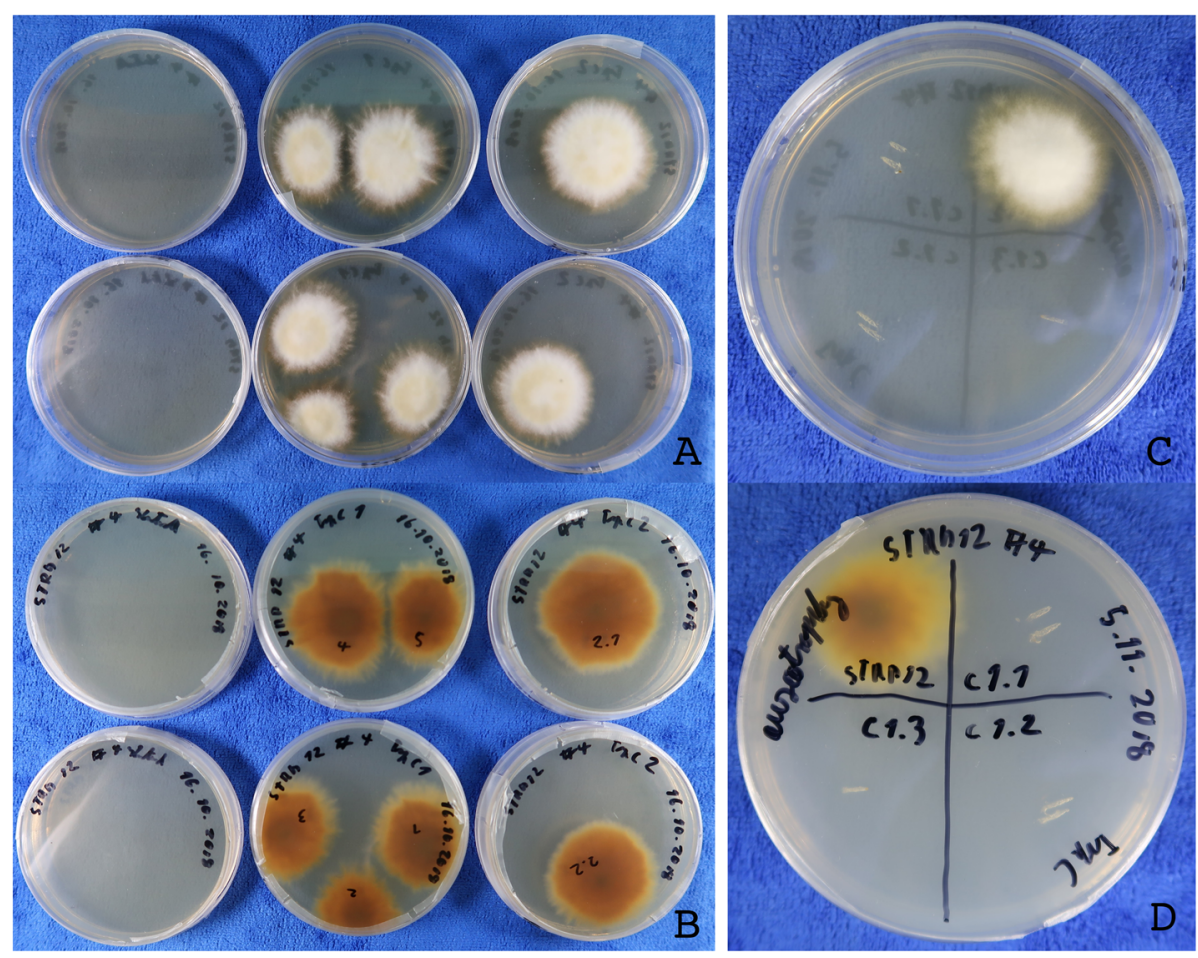

Fig. 5 Transformation and analysis of T. rubrum $\Delta$ trp3. a and $\mathbf{b}$ One month old transformation plates. Control plates (left) without colonies, transformation with sgRNA construct 1 (middle) and transformation with sgRNA construct 2 (right). $\mathbf{c}$ and $\mathbf{d}$. Auxotrophy test of strain 1-3 of construct 1 on SC medium without tryptophan. The wild-type strain STRB012 grown well whereas $\Delta$ trp3 strains $\mathrm{C} 1.1-\mathrm{c} 1.3$ are auxotrophic for tryptophan 
sequenced strains were 2380 resp. $1955 \mathrm{bp}$. In one strain an additional nucleotide had been inserted between the inserted Ura3 fragment and the theoretical Cas9 blunt end restriction site. Besides the mutations at the cloning site, neither the inserted fragments nor the flanking regions had further mutations.

\section{Discussion}

We have set up a transformation system for the obligate human pathogenic fungus T. rubrum. For this, we had isolated the strain STRB012, which is suitable for routine genetic transformations. Since the transformation protocol is based on creating protoplast from microconidia, we had chosen a strain with a high rate of conidiogenesis. As in other T. rubrum strains, the conidiogenesis can even be enhanced by increasing the $\mathrm{CO}_{2}$ concentration in the atmosphere [9]. To prevent genetic changes by serial transfer, the procedure from isolation, via monoclonal isolation until freeze-stock has been kept as short as possible [10]. Moreover, to ensure homogeneous genetic material, the freeze-stock had been aliquot. So far, the selection of transformed Trichophyton strains was based on drug resistances genes, mostly the hygromycin B resistances gene. Thereby, most of the genetic manipulations in the genus Trichophyton were performed with hygromycin B susceptible T. mentagrophytes strains. For example, the growth of T. mentagrophytes CBS 570.80 was completely inhibited at a concentration of $20 \mu \mathrm{g} / \mathrm{ml}$ and selected at a final concentration of $100 \mu \mathrm{g} / \mathrm{ml}$ hygromycin B [11]. Mutations of $T$. mentagrophytes TIMM2789 were selected with a concentration of $250 \mu \mathrm{g} / \mathrm{ml}$ hygromycin B in the overlay medium [12]. On the other hand, T. rubrum CBS118892 has a 10 times higher tolerance for hygromycin B with a minimum inhibitory concentration (MIC) of $200 \mu \mathrm{g} / \mathrm{ml}$ for microconidia and $800 \mu \mathrm{g} / \mathrm{ml}$ for arthroconidia [13]. Besides this, the selection with auxotrophic markers has the advantage of being more stringent and $\Delta$ ura3 strain do not grow in the absence of uracil.

For creation of uracil auxotrophic strains, we first predicted, which gene in the genome of T. rubrum could be homologous to the Ura3 gene of S. cerevisiae. By knocking out this gene, we resulted uracil auxotrophic strains and used for the counter selection $1 \mathrm{~g} / \mathrm{l} \mathrm{FOA}$. Whereas this concentration in yeast is sufficient, the T. rubrum wild-type exhibited slow growth and had been visible as downy hyphae. Since the transformed strains were visible as distinct colonies, they were isolated by inoculating to fresh FOA plates. To proof undoubtfully, that the mutation in the URA3 gene is the cause of auxotrophy, we recovered the function by knock in the URA3 gene. For this, we chose the TRP3 gene as a target and changed the genotype from $\triangle U R A 3, T R P 3$ into $\triangle T R P 3, U R A 3$.
We have demonstrated that gene knock-outs in $T$. rubrum can be created with Cas9 in two ways. First, by inducing DSBs with a Cas9 ribonucleoprotein complex. In the process from inducing the DSB until the NHEJ repair, with a certain likelihood, mutations occurs. In S. cerevisiae a significant fraction of the mutations were +1 indels caused by 1 bp $5^{\prime}$ overhangs at the Cas 9 cleavage sites. Thereby most of the +1 indels required the DNA polymerase Pol4 to fill in $5^{\prime}$ overhangs [14]. These +1 indels are causing frame shifts in the gene translation and are disabling the protein function. Second, by inducing DSBs and integration of a selection marker. Since the URA3 selection marker has several stop codons in all 6 reading frames, the translation will be interrupted and the knockout strains could be selected by using the uracil selection. In both cases the DSBs had been repaired by NHEJ.

In Aspergillus nidulans both NHEJ and HDR pathways are engaged in repairing Cas9 induced DNA DSBs. A NHEJ proficient $A$. nidulans strain could be transformed with Cas9 without supplying homologous DNA fragments, whereas NHEJ deficient strains required genetargeting substrates (GTS) for efficient transformation. The GTSs had homologous sequences to both flanking sites of the DSBs whereby the highest efficiencies had circular GTSs with 1000 - 2000 bp homologous sequences. Interestingly, also single-stranded DNA fragments with a length of $90 \mathrm{bp}$ induced the HDR mechanism [15]. Whereas in Trichophyton and Aspergillus spp. the NHEJ is dominant, in S. cerevisiae quasi all DSBs are repaired by HDR in the presence of a homologous template [16]. Also in Candida glabrata only a modest increase in targeting efficiency (1.5\% vs $1.1 \%$ ) was seen in NHEJ deficient strain compared to the wild-type [17].

Genetic manipulations of $S$. cerevisiae are now performed for more than 40 years and many tools and protocols have been developed in this time. Nowadays, genetic manipulations of $S$. cerevisiae, and as well of $C$. albicans, are routines, whereas transformations of non-model fungal species are still often challenging. We developed a transformation protocol for such a species and are convinced, that the protocol can be adapted to other fungal species. With the development of NGS and bioinformatics tools, it is now possible to predict the URA3 gene sequence of nearly every fungal strain with reasonable effort. The developed protocol is not depending on further species specific tools as autonomous replication vectors. We are convinced that with further developments of the NGS in combination with the Cas9 technique the knowledge of the genetics of non-model fungal species will increase significantly in the near future.

\section{Conclusions}

In fungal model organism, gene knock-out are routinely performed by using an integrating selection marker, 
which allows selection against the auxotrophic parent strain, into the target gene. We developed for the first time a protocol, based on in vitro synthesized Cas9sgRNA ribonucleoprotein complex, to adapt the well established method to non-model fungal species. We knocked out the Ura3 gene in T. rubrum and used this strain for target specific gene knock-outs. The gene knock-out of the Ura3 gene as well of the Trp3 gene led to auxotrophy in T. rubrum.

\section{Materials and methods Fungal strains}

The fungal strains STRB008 and STRB012 were isolated at the Institute of Dermatology in Nanjing, China. Both strains were isolated by the clinical personal of the hospital for diagnosis and treatment purpose. Strain STRB008 was isolated in June 2017 from a foot nail and strain STRB012 was isolated in July 2017 from the leg of a patient. All created knock-out strains were derived from clinical isolate STRB012 except of the strain STRB020, which was a descendant of clinical isolate STRB008 (Table 2).

\section{PCR and vector}

PCR reactions were performed in 25 or $50 \mu \mathrm{l}$ using Pfu polymerase (Vazyme, China, Cat\#P505) with an initial denaturation step at $95^{\circ} \mathrm{C}$ for $2 \mathrm{~min}, 30$ to 35 cycles of denaturation for $45 \mathrm{~s}$, annealing at $50-60{ }^{\circ} \mathrm{C}$ for $30 \mathrm{~s}$ and elongation at $72^{\circ} \mathrm{C}$ for $1 \mathrm{~min}$ per $1 \mathrm{~kb}$. The finalization at $72^{\circ} \mathrm{C}$ had a duration of $1-2$ times of the elongation. Vectors (Table 3) were constructed by using the pUC19 backbone and manipulated with the Mutagenesis Kit (Vazyme, \#C215) or by sticky end restriction and T4 DNA ligation. Plasmids were amplified in E. coli DH5a and purified using a Plasmid isolation kit (Qiagen, Germany, \#27106).

For the construction of the plasmid PTRB024, the URA3 gene had been amplified from strain STRB012 with the primer pair NTRB060/61 and restricted with EcoRI/BamHI. The cloning vector had been restricted

Table 2 T. rubrum strains

\begin{tabular}{|c|c|c|c|}
\hline Strain & Parent strain & Phenotype & Genotype \\
\hline STRB008 $^{*}$ & & WT & WT \\
\hline STRB012* & & WT & WT \\
\hline STRB018 & STRB012 & $\mathrm{Ura}^{-}$ & ura3- $\Delta 363^{* *}$ \\
\hline STRB019* & STRB012 & $\mathrm{Ura}^{-}$ & ura3-363_364insA \\
\hline STRB020* & STRB008 & Ura $^{-}$ & \\
\hline$\Delta \operatorname{Trp} \_c 1.1$ & STRB018 & $\operatorname{Trp}^{-}$ & ura3- $\triangle 363, \operatorname{trp3}:: U R A 3^{* *}$ \\
\hline$\Delta \operatorname{Trp} \_c 1.2$ & STRB018 & $\operatorname{Trp}^{-}$ & ura3- $\triangle 363, \operatorname{trp} 3:: U R A 3^{* *}$ \\
\hline
\end{tabular}

*preserved at the Chinese National Medical Fungal Collection in Nanjing **verified by sequencing
Table 3 Plasmids

\begin{tabular}{ll}
\hline Plasmid $^{*}$ & Insert \\
\hline PTRB024 & $\begin{array}{l}\text { orotidine 5-phosphate decarboxylase } \\
\text { ('Ura3') with flanking regions }\end{array}$ \\
PTRB025 & $\begin{array}{l}\text { template plasmid to synthesis Ura3 } \\
\text { sgRNA } \\
\text { anthranilate synthase component 2 } \\
\text { ('Trp3)' with flanking regions }\end{array}$ \\
PTRB035 & $\begin{array}{l}\text { template plasmid to synthesis Trp3 } \\
\text { sgRNA }\end{array}$ \\
PTRB042 & template plasmid to synthesis Trp3 sgRNA \\
PTRB043 &
\end{tabular}

"The complete sequences of the plasmids, including annotation, are given in the Additional file 1

and dephosphorylated before inserting the URA3 fragment.

We constructed a vector, PTRB025, containing the sgRNA fragment of pFC334 (Addgene, USA, \#87846) [18], the T7 promoter and a protospacer unique for the T. rubrum URA3 gene. Afterwards, the fragment was amplified with the primer pair NTRB80/81, agarose gel extracted and used as template for the sgRNA transcription. The transcription had been performed with a T7 RNA polymerase (Vazyme, \#TR101) according to the producer manual. We used $500 \mathrm{ng}$ of the template DNA and incubated for $4 \mathrm{~h}$ at $37^{\circ} \mathrm{C}$. Afterwards, the transcript was purified with a RNA Clean-Up Kit (Takara, Japan, \#632638).

After purification, $4 \mu \mathrm{g}$ sgRNA was mixed with $12 \mu \mathrm{g}$ Cas9 protein (Takara, \#632641) in $30 \mu \mathrm{l} \mathrm{TE}$ buffer and incubated for $5 \mathrm{~min}$ at $37^{\circ} \mathrm{C}$ to induced complex formation. After adding $5 \mu \mathrm{l}$ of a $50 \mathrm{mMol}$ spermidine (Sigma-Aldrich, USA, \#S0266) solution, the transformation solution was stored at $-80^{\circ} \mathrm{C}$ until use. The plasmid PTRB042 and 43 were constructed by exchanging the protospacer region of PTR025 from URA3 to TRP3 homologous by using the primer pairs NTRB107/108 resp. 109/110. The sgRNA was transcribed from linearized DNA fragments of PTRB042 ('construct 1') and 43 ('construct 2'). For the Uracil selection, $3 \mu \mathrm{g} U R A 3$ fragments with flanking regions were amplified from plasmid PTRB024 with the primer pair NTRB85/86.

\section{Transformation}

For the transformation, $\mathrm{KCl}$ buffer $(1 \mathrm{M} \mathrm{KCl}$ in $10 \mathrm{mMol}$ sodium phosphate buffer, pH 5.8), lysis buffer ( $\mathrm{KCl}$ buffer with $10 \mathrm{mg} / \mathrm{ml}$ lysing enzymes (Sigma-Aldrich, \#L1412) and driselase (Sigma-Aldrich, \#D9515); stored at $\left.-20^{\circ} \mathrm{C}\right)$, sucrose buffer $(1 \mathrm{M}$ sucrose, $10 \mathrm{mMol}$ Tris $\left.\mathrm{HCl} \mathrm{pH} \mathrm{7.5,} 10 \mathrm{mMol} \mathrm{CaCl}_{2}\right)$ and a PEG buffer $(60 \%$ (w/v) PEG 4000, $10 \mathrm{mMol}$ Tris $\mathrm{HCl} \mathrm{pH} \mathrm{7.5,} 10 \mathrm{mMol}$ $\mathrm{CaCl}_{2}$ ) were prepared.

The overlay media were based on SC medium (see Additional file 2) supplemented with $1 \mathrm{M}$ sucrose and 
1\% low melting agarose (Sigma-Aldrich, \#A9045). The media were melted at $80^{\circ} \mathrm{C}$ and cooled down to $40^{\circ} \mathrm{C}$ before adding the protoplasts. For the FOA counterselection, the media have been adjusted to $\mathrm{pH} 5$ and $1 \mathrm{~g} / \mathrm{l}$ FOA (Zymo Research, USA, \#F9001) were added.

\section{Sequencing}

For Next Generation Sequencing (NGS), $0.5 \mathrm{~g}$ mycel from 10 days old liquid cultures were harvested and disrupted in liquid nitrogen. DNA was extracted using Qiagen plant extraction kit. The fragments, approximate $400 \mathrm{bp}$ length, were pair-end sequenced with read length of $150 \mathrm{bp}$ using the Hiseq 4000 platform. The sequenced fragments were mapped with Bowtie 2 [19] and aligned with Samtools [20] to the reference genome T. rubrum CBS 118892 (ASM15142v1). The alignments of the Fastq files to the reference assembly was visualized with Consed [21] and the regions of interest were analyzed for polymorphisms before cloning and primer designing. The ITS1 and 2 sequences were extracted from the NGS data and aligned with ClustalX.

\section{Supplementary information}

Supplementary information accompanies this paper at https://doi.org/10. 1186/s12896-020-0601-z.

Additional file 1. Supplement_sequences; DNA sequences of plasmids, primers and ITS 1 and 2 including alignment.

Additional file 2. Supplement_media; cultivation media.

\section{Abbreviations}

Cas9: Protein of the CRISPR/Cas9 system; DSB: Double string break in a chromosome; FOA: 5-fluoroorotic acid; HDR: Homologous directed repair of a chromosome; NHEJ: Non-homologous end joining of a chromosome; NTRBOXX: Nucleic acid and primers; PTRBOXX: Plasmids; SC: Synthetic Complete medium; sgRNA: Small guided RNA of the Cas9-sgRNA ribonucleoprotein complex; STRBOXX: T. rubrum strain; Tr1: Trichophyton cultivation medium Nr. 1; TRP3: Anthranilate synthase component 2 gene; URA3: Orotidine-5'-monophosphate decarboxylase gene

\section{Acknowledgements}

Not Applicable.

\section{Authors' contributions}

All authors were involved in conceiving and designing the study. OB was involved in all parts of the study. HM organized the resources and laboratory. XZ performed knock-out experiments and plasmid constructions. HZ provided the fungal strains, plasmids, preserves the strains and were involved in fungal transformations. GL and WL acquired the fundings. All authors have read and approved the manuscript.

\section{Funding}

This study was supported by CAMS Innovation Fund for Medical Science (CAMS-I2M,2016-I2M-3-021), the National Natural Science Foundation of China (No.819272949), Sub-Item of the Important and Special Project of the Science and Technology Ministry of China (No.2018ZX10734404) and the Basical Scientific Research Fund Projects of Chinese Academy of Medical Sciences (No.2018PT31013). The funders had no role in study design, data collection, analysis, decision to publish or preparation of the manuscript.
Availability of data and materials

The datasets used and/or analysed during the current study are available from the corresponding author on reasonable request.

Ethics approval and consent to participate

We declare that no permission were necessary for the specimens used in our study.

\section{Consent for publication}

Not Applicable.

\section{Competing interests}

The authors reports no conflict of interest.

\section{Author details}

'Department of Medical Mycology, Institute of Dermatology, Chinese Academy of Medical Science and Peking Union Medical College, Nanjing, Jiangsu 210042, People's Republic of China. ${ }^{2}$ Jiangsu Key Laboratory of Molecular Biology for Skin Diseases and STIs, Nanjing, Jiangsu 210042, People's Republic of China. ${ }^{3}$ Center for Global Health, School of Public Health, Nanjing Medical University, Nanjing, Jiangsu 211166, People's Republic of China.

Received: 21 August 2019 Accepted: 14 January 2020

Published online: 20 January 2020

\section{References}

1. Alshahni MM, Yamada T. Genetic manipulations in Dermatophytes. Mycopathologia. 2017;182:33-43.

2. Boeke JD, Lacroute F, Fink GR. A positive selection for mutants lacking orotidine-5'-phosphate decarboxylase activity in yeast: 5-fluoro-orotic acid resistance. Mol Gen Genet. 1984 197: 345-346.

3. Kaufman G, Horwitz BA, Hadar R, Ullmann Y, Berdicevsky I. Green fluorescent protein (GFP) as a vital marker for pathogenic development of the dermatophyte Trichophyton mentagrophytes. Microbiology. 2004;150: 2785-90.

4. Yamada T, Makimura K, Hisajima T, Ishihara Y, Umeda Y, Abe S. Enhanced gene replacements in Ku80 disruption mutants of the dermatophyte, Trichophyton mentagrophytes. FEMS Microbiol Lett. 2009:298:208-17.

5. Wu WY, Lebbink JHG, Kanaar R, Geijsen N, van der Oost J. Genome editing by natural and engineered CRISPR-associated nucleases. Nat Chem Biol. 2018;14:642-51.

6. Zhang C, Meng X, Wei X, Lu L. Highly efficient CRISPR mutagenesis by microhomology-mediated end joining in Aspergillus fumigatus. Fungal Genet Biol. 2016;86:47-57.

7. Nagy G, Szebenyi C, Csernetics A, Vaz AG, Tóth EJ, Vágvölgyi C, Papp T. Development of a plasmid free CRISPR-Cas9 system for the genetic modification of Mucor circinelloides. Sci Rep. 2017;7:16800.

8. Wang Q, Cobine PA, Coleman JJ. Efficient genome editing in Fusarium oxysporum based on CRISPR/Cas9 ribonucleoprotein complexes. Fungal Genet Biol. 2018:117:21-9.

9. Laurent A, Monod M. Production of Trichophyton rubrum microspores in large quantities and its application to evaluate amorolfine/azole compound interactions in vitro. Mycoses. 2017;60:581-6.

10. Homolka L. Preservation of live cultures of basidiomycetes - recent methods. Fungal Biol. 2014;118:107-25.

11. Gonzalez R, Ferrer S, Buesa J, Ramon D. Transformation of the dermatophyte Trichophyton mentagrophytes to hygromycin B resistance. Infect Immun. 1989:57:2923-5.

12. Yamada T, Makimura K, Uchida K, Yamaguchi H. Reproducible genetic transformation system for two dermatophytes, Microsporum canis and Trichophyton mentagrophytes. Med Mycol. 2005:43:533-44.

13. Coelho LM, Aquino-Ferreira R, Maffei CM, Martinez-Rossi NM. In vitro antifungal drug susceptibilities of dermatophytes microconidia and arthroconidia. J Antimicrob Chemother. 2008;62:758-61.

14. Lemos BR, Kaplan AC, Bae JE, Ferrazzoli AE, Kuo J, Anand RP, Waterman DP, Haber JE. CRISPR/Cas9 cleavages in budding yeast reveal templated insertions and strand-specific insertion/deletion profiles. Proc Natl Acad Sci U S A. 2018;115:E2040-7. 
15. Nødvig CS, Hoof JB, Kogle ME, Jarczynska ZD, Lehmbeck J, Klitgaard DK, Mortensen UH. Efficient oligo nucleotide mediated CRISPR-Cas9 gene editing in Aspergilli. Fungal Genet Biol. 2018;115:78-89.

16. Hinnen A, Hicks JB, Fink GR. Transformation of yeast. Proc Natl Acad Sci U S A. 1978;75:1929-33.

17. Cen Y, Timmermans B, Souffriau B, Thevelein JM, Van Dijck P. Comparison of genome engineering using the CRISPR-Cas9 system in C. glabrata wild-type and lig4 strains. Fungal Genet Biol. 2017;107:44-50.

18. Nødvig CS, Nielsen JB, Kogle ME, Mortensen UH. A CRISPR-Cas9 system for genetic engineering of filamentous Fungi. PLoS One. 2015;10:1-18.

19. Langmead B, Salzberg S. Fast gapped-read alignment with bowtie 2. Nat Methods. 2012;9:357-9.

20. Li H, Handsaker B, Wysoker A, Fennell T, Ruan J, Homer N, Marth G, Abecasis G, Durbin R. 1000 genome project data processing subgroup. The sequence alignment/map (SAM) format and SAMtools. Bioinformatics. 2009;25:2078-9.

21. Gordon D, Green P. Consed: a graphical editor for next-generation sequencing. Bioinformatics. 2013;29:2936-7.

\section{Publisher's Note}

Springer Nature remains neutral with regard to jurisdictional claims in published maps and institutional affiliations.

Ready to submit your research? Choose BMC and benefit from:

- fast, convenient online submission

- thorough peer review by experienced researchers in your field

- rapid publication on acceptance

- support for research data, including large and complex data types

- gold Open Access which fosters wider collaboration and increased citations

- maximum visibility for your research: over $100 \mathrm{M}$ website views per year

At BMC, research is always in progress.

Learn more biomedcentral.com/submissions 